\title{
EVERY REAL SYMPLECTIC MATRIX IS A PRODUCT OF COMMUTATORS OF REAL SYMPLECTIC INVOLUTIONS
}

\author{
XIN Hou
}

Abstract. Denote by $I\left(I_{n}\right)$ the $(n \times n)$ identity matrix. A matrix $A$ is symplectic if $A^{T} J A=J$, where $J=\left[\begin{array}{cc}0 & I_{n} \\ -I_{n} & 0\end{array}\right]$. A symplectic matrix $A$ is a commutator of symplectic involutions if $A=$ $X Y X^{-1} Y^{-1}$, where $X$ and $Y$ are symplectic and $X^{2}=Y^{2}=I$. Let $\mathbb{R}$ be the real number field. Denote by $\operatorname{tr} A$ the trace of $A$, by $A \oplus B$ the direct sum of $A$ and $B$. In this article, it is proved that every $4 \times 4$ real symplectic matrix except the matrices similar to $-I_{2} \oplus B$ for $-I_{2} \neq B \in \operatorname{Sp}(2, \mathbb{R})$ and $\operatorname{tr} B \geqslant-2$, can be decomposed into a product of at most two commutators of real symplectic involutions, and the exceptional real symplectic matrices are products of three commutators of real symplectic involutions. Using this result, it is shown that every real symplectic matrix of size greater than two is a product of a finite number of commutators of real symplectic involutions.

Mathematics subject classification (2020): 15A23, 20H25, $20 \mathrm{H} 20$.

Keywords and phrases: Involutions, commutators of involutions, symplectic matrices.

\section{REFERENCES}

[1] D. AWA, R. J. DE LA CRUZ, Every real symplectic matrix is a product of real symplectic involutions, Linear Algebra Appl. 589 (2020) 85-95.

[2] R. J. DE LA CRUZ, Each symplectic matrix is a product of four symplectic involutions, Linear Algebra Appl. 466 (2015) 382-400.

[3] E. Ellers, J. Malzan, Products of positive transvections in the real symplectic group, Comm. Algebra 19 (4) (1991) 1033-1058.

[4] E. W. Ellers, O. Villa, Generation of the symplectic group by involutions, Linear Algebra Appl. 591 (2020) 154-159.

[5] W. H. Gustafson, P. R. Halmos, H. Radjavi, Products of involutions, Linear Algebra Appl. 13 (1976) 157-162.

[6] J. GUTT, Normal forms for symplectic matrices, http://arxiv.org/abs/1307. $2403 v 2$.

[7] X. Hou, Decomposition of infinite matrices into products of commutators of involutions, Linear Algebra Appl. 563 (2019) 231-239.

[8] X. Hou, Decomposition of symplectic matrices into products of commutators of symplectic involutions, Comm Algebra. 48 (8) (2020) 3459-3470.

[9] X. HoU, Products of commutators of symplectic involutions, Linear Multilinear Algebra, https://doi.org/10.1080/03081087.2020.1820432.

[10] X. Hou, S. LI, Q. ZHENG, Expressing infinite matrices over ring as products of involutions, Linear Algebra Appl. 532 (2017) 257-265.

[11] B. HUPPERT, Isometrien von Vektorraumen I, Arch. Math. 35 (1980) 164-176.

[12] T. J. LAFFEY, Expressing unipotent matrices over rings as products of involutions, Irish Math. Soc. Bull. 40 (1998) 24-30.

[13] K. M. LiU, Decomposition of matrices into three involutions, Linear Algebra Appl. 111 (1988) 1-24.

[14] R. SŁOWIK, Expressing infinite matrices as products of involutions, Linear Algebra Appl. 438 (2013) 399-404. 
[15] B. ZHEnG, Decomposition of matrices into commutators of involutions, Linear Algebra Appl. 347 (2002) 1-7. 